\title{
Questioning speech acts ${ }^{1}$
}

Jess H.-K. LAW - Rutgers University

Haoze LI - New York University

Diti BHADRA - Harvard University

\begin{abstract}
We investigate the sentence-final particle ho from Cantonese, which can stack on top of other sentence-final particles indicating various types of speech acts. We argue that ho is a higher level question operator that operates at the level of speech acts. More concretely, it takes a speech act (assertion or question) and returns a new interrogative speech act asking whether the input speech act can be felicitously performed by the addressee. We take the presence of this kind of higher level question operator in natural language as novel evidence that a mechanism for operating on speech acts is needed. Building on Farkas and Bruce (2009), Rawlins (2010), Bledin and Rawlins (2017), we develop a mechanism in the style of Update Semantics for operating on speech acts.
\end{abstract}

Keywords: speech acts, sentence-final particles, Cantonese, update semantics.

\section{Introduction}

The function of mapping from the semantic content of an utterance to its convention of use (a division of labor first made by Frege (1956)) has been attributed to abstract speech act operators (also known as force operators), such as ASSERT, QUESTION, and COMMAND. These operators have been traditionally assumed to occupy the highest echelons of the clausal periphery. The precise formulation of these operators has attracted a lot of attention from semanticists, as they are crucial for formalizing the diverse discourse functions of speech acts (Farkas and Bruce, 2009; Farkas and Roelofsen, 2017; Malamud and Stepheson, 2015; Krifka, 2015). These high operators usually come packaged with two assumptions: i) they are not embeddable under other elements, and ii) they belong to the realm of pure pragmatics and not compositional semantics. Recent research in both semantics and syntax have challenged these assumptions (Krifka, 2015; Davis, 2011; Wiltschko, 2017; Heim et al., 2016). Based on evidence from a language with a rich array of sentence-final particles (SFPs), Cantonese, we argue in this paper that not only are abstract speech operators embeddable, it is also the case that we need compositional mechanisms in these high regions of the clause. We will investigate the SFP stacking phenomenon, and argue that such grammaticalized operations on speech act operators reveal the need for a system that can compose the content of an utterance with multiple particles that update the discourse in a number of different, non-trivial ways.

\footnotetext{
${ }^{1}$ We would like to thank Justin Bledin, Kyle Rawlins, Manfred Krifka, Sze-Wing Tang, Veneeta Dayal and audiences at SuB-22 and NELS-48 for their comments and suggestions.
} 


\section{The empirical landscape}

\subsection{Primary sentence-final particles in Cantonese}

Cantonese is a language with a large repository of sentence-final particles. These sentence-final particles are standardly taken to be elements that serve the myriad functions that various intonational contours serve in Indo-European languages such as English (Wakefield, 2011). As such, one of their roles is to indicate illocutionary force, or speech acts (Cheung, 1972; Luke, 1990; Matthews and Yip, 2011; Fung, 2000). Note that different sentence-final particles may indicate the same speech act with slightly different flavors. Since the purpose of this subsection is to introduce the basic particles to be taken up later, we only include a small set of particles that we will use later. $^{2}$ To express an assertion, the particle gaa may be used, as shown in (1).

$$
\begin{aligned}
& \text { Aaman sik haa gaa. } \\
& \text { Aaman eat shrimp ASSERT } \\
& \text { 'Aaman eats shrimp.' }
\end{aligned}
$$

Assertion

When expressing an interrogative, one may choose from a range of sentence-final particles, depending on the type of the interrogative. For example, a polar question may be accompanied by the particle maa:

$$
\begin{aligned}
& \text { Aaman sik haa maa? } \\
& \text { Aaman eat shrimp POLQ } \\
& \text { 'Does Aaman eat shrimp?' }
\end{aligned}
$$

Polar question

To mark a wh-question or an alternative question, ne may be used, as shown in (3a) and (3b), respectively.

$$
\begin{aligned}
& \text { a. Bingo sik haa ne? } \\
& \text { who eat shrimp wHQ } \\
& \text { 'Who eats shrimp?' } \\
& \text { b. Aaman sik haa ding sik ju ne? } \\
& \text { Aaman eat shrimp or eat fish WHQ } \\
& \text { 'Does Aaman eat shrimp or fish?' }
\end{aligned}
$$

Wh-question

Alternative question

It is worth noting that sentence-final particles in general are an optional device to mark clause types. Strictly speaking, one can still get the intended clause type without using any sentence-final particle, especially in a more formal speech context or a written context. However, native speakers feel that having sentence-final particles helps make utterances more natural.

\footnotetext{
${ }^{2}$ We gloss this set of basic particles based on the type of speech acts they indicate, such as ASSERT for assertive particles, POLQ for polar question particles, and WHQ for wh-question and alternative question particles. All other sentence-final particles that are not the concern of this paper are glossed as SFP.
} 
Now that we have acquainted ourselves with the primary sentence-final particles in Cantonese, we are ready to turn to a particle that may stack on top of a primary particle. The particle of interest is ho. We introduce ho's interactions with different speech acts: with assertions in section 2.2, and with questions in 2.3 .

\subsection{Ho embedding assertions}

$\mathrm{Ho}$ is an interrogative sentence-final particle in Cantonese. It is special because it may stack on top of another sentence-final particle, as shown in (4).

(4) Aaman sik haa gaa ho?

Aaman eat shrimp ASSERT HO

'Aaman eats shrimp. Right?'

Assertion + ho

In this example, ho stacks on the assertion particle gaa. It has the effect of turning the assertion into a question, as pointed out in previous studies (Sybesma and Li, 2006; Lam, 2014; Tang, 2015). Following Lam (2014), this type of questions is roughly translated as an assertion plus a confirmation tag 'right?'. However, this is by no means a commitment to equating Cantonese ho and the English confirmation tag.

An assertion +ho question admits a range of responses also admitted by an ordinary polar question. For example, one may choose an affirmative answer like (5a), a negative answer like (5b), or indicate their ignorance with $(5 \mathrm{c})$.
a. Hai aa.
b. Mhai aa
no SFP
'No, he doesn't'
'Yes, he does.'
c. jo mzi wo
I not.know SFP
'I don't know.'

At this point, one may be tempted to analyze $h o$ as a polar question marker similar to maa. However, these two particles exhibit crucial differences with respect to their ability to stack on a primary particle. Observe that unlike ho, the polar question particle maa may not stack on an assertion particle, as evidenced by the unacceptability of (6).

$$
\begin{aligned}
& \text { *Aaman sik haa gaa maa? } \\
& \text { Aaman eat shrimp ASSERT POLQ } \\
& \text { Intended 'Does Aaman eat shrimp?' }
\end{aligned}
$$

We take the difference in stackability to be semantically grounded. While ordinary question parti-

\footnotetext{
${ }^{3}$ Gaamaa may be used as a complex assertive particle to indicate obviousness of the asserted content. In this case, it is a fusion of two assertive particles ge and amaa, rather than a fusion of an assertive particle and a polar question particle, as suggested by Matthews and Yip (2011).
} 
cles like maa signal the mapping from semantic content to interrogative speech act, ho is a 'higher level' question particle embedding speech act rather than just a semantic content. If this view is correct, then the ungrammaticality of (6) is expected, as maa indicates a transition from semantic content to a speech act - the input is already a speech act, as indicated by the presence of a sentence-final particle, so, in a sense, it is 'too big' to be operated on by maa. By contrast, ho is perfectly happy to operate on a unit already marked by a sentence-final particle, as shown in (4), because it is a 'higher level' question particle, one that takes a speech act as its input. We will undertake a formalization of the property of being a 'higher level' question operator in Section 3.3.

In addition, when ho takes an assertive speech act as its input, it indicates a bias towards the asserted content, another trait not shared by the polar question particle maa. Imagine a neutral context in which someone is trying to ask a pedestrian to fill out a survey. (7) may be used felicitously to make such a request, but (8) may not.

$$
\begin{aligned}
& \text { Nei jau sigaan maa? } \\
& \text { you have time POLQ } \\
& \text { 'Do you have time?' }
\end{aligned}
$$

Polar question

$$
\begin{aligned}
& \text { \#Nei jau sigaan gaa ho? } \\
& \text { you have time ASSERT HO } \\
& \text { 'You have time. Right?' }
\end{aligned}
$$

Assertion + ho

If (8) is used, it conveys the message that the speaker has prior belief that the addressee has time to help, which comes across as impolite in this context. On the contrary, (7) does not have such a bias, so it does not have connotations of impoliteness in the same context.

\subsection{Ho embedding questions}

An even more interesting distributional fact is that ho may readily embed a $w h$-question or an alternative question (Lam, 2014). ${ }^{4}$ Consider the following examples:

(9) Bingo sik haa ne ho?

who eat shrimp WHQ HO

'Who eats shrimp? Do you share the same question?' Wh-question + ho

(10) Aaman sik haa ding sik jyu ne ho?

Aaman eat shrimp or eat fish WHQ HO

'Does Aaman eat shrimp or fish? Do you share the same question?' Alt question + ho

${ }^{4}$ Ho may also embed other types of questions, but a more sophisticated context is required. We discuss this issue in Section 4. 
When stacked on top of a question particle, as in (9)-(10), ho changes the question introduced by the lower question particle into another question asking roughly whether the addressee would rationally ask the same embedded question, irrespective of the type of the question. Since using a confirmation tag after a question does not sound felicitous to native speakers of English, we chose to translate the contribution of ho in interrogative cases by using another question, i.e., 'do you share the same question?'

We would like to point out two notable facts about question-ho constructions. First, the range of responses this construction admits is quite different from the ones admitted by just using the embedded questions. Consider some responses to a wh-question like (11), illustrated in (12a)$(12 \mathrm{c})$.

$$
\begin{aligned}
& \text { Bingo sik haa ne? } \\
& \text { who eat shrimp WHQ } \\
& \text { 'Who eats shrimp?' }
\end{aligned}
$$
a. Aaman lo.
b. jo mzi wo.
I not.know SFP
'I don't know.'
c. \#Hai lo.
yes SFP
'Aaman S
'Yes.'

If one knows the answer to (11), they may directly answer it, as in (12a). Alternatively, if one does not know the answer, they may indicate their ignorance with (12b). However, one may not answer hai (lo) 'yes' to such a question. ${ }^{5}$

After such a wh-question is embedded under ho, as in (13), the range of felicitous responses changes, as shown in (14a) - (14d).

Bingo sik haa ne ho?

who eat shrimp WHQ HO

'Who eats shrimp? Do you share the same question?'
a. Aaman lo.
c. no dou mzi wo.
I also not.know SFP
Aaman SFP
'Aaman.'
b. \#no mzi wo.
I not.know SFP
'I don't know.'
'I don't know either.'
d. Hai lo.
yes SFP
'Yes.'

\footnotetext{
${ }_{5}^{5}$ Hai (lo) 'yes' may be used when a continuation like I don't know either is added. Anticipating the discussion of ho stacking on top of a question, which readily admits such as response without the need of a continuation like I don't know either, we suggest that a canonical question may be turned into a higher, speech act-level question via some pragmatic means. The continuation can be seen as a trigger of the pragmatic means.
} 
It is still possible to directly answer the wh-question, as shown in (14a). However, it is no longer felicitous to just answer I don't know, as shown in (14b). To indicate ignorance, the addressee has to show agreement with the speaker's ignorance, by using a response corresponding to I don't know either, as shown in (14c). Moreover, rather surprisingly, it is felicitous to answer hai (lo) 'yes', as shown in (14d).

Secondly, when ho embeds a question, it changes the felicity condition associated with the embedded question. Generally speaking, a speaker uses an interrogative speech act to signal the belief that the addressee may be able to answer the question. However, a speaker signals just the opposite when he or she uses ho to embed an interrogative speech act. That is, the speaker thinks it is possible that the addressee may not be able to answer the embedded question. We illustrate the contrast in the two types of questions with two storyboard scenarios borrowed from the UBC Syntax of Speech Acts Lab.

Scenario A: 'My friend was puzzled, too.'
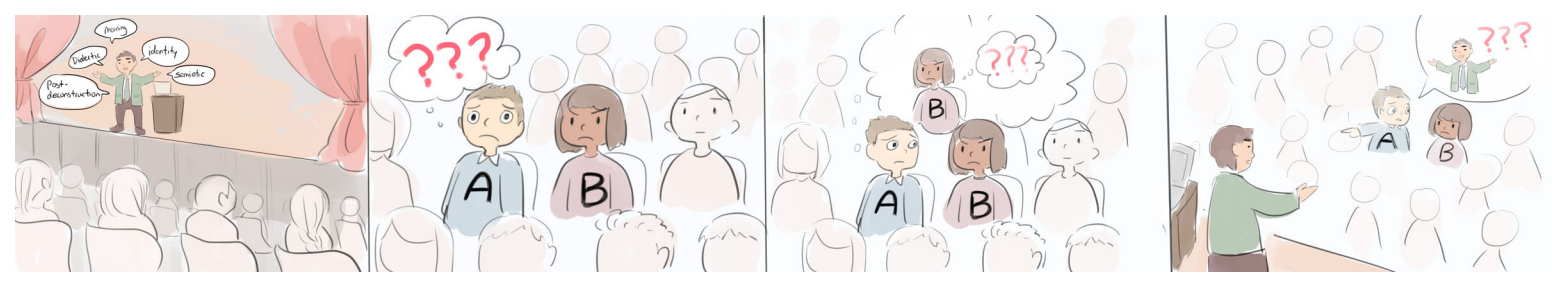

Figure 1: A famous scientist gave a talk on astrophysics. A, as a linguist, couldn't follow the talk. A's friend B was a poet, and it seemed to A that B did not understand the talk either.

Scenario B: 'My friend understood this.'

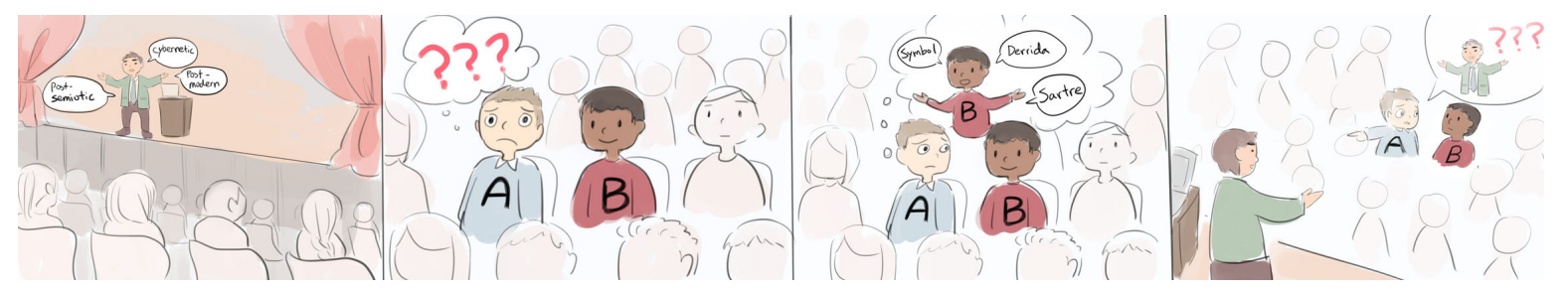

Figure 2: A famous scientist gave a talk on astrophysics. A, as a linguist, cannot follow the talk. However, A's friend B was a physicist and it seemed to A that B understood the talk quite well.

In the first scenario, A did not understand the content of the talk and thought that B did not understand it either. In this context, using a wh-question+ho like (16) is felt to be more felicitous than using a wh-question like (15), if A did not have obnoxious intentions. If $\mathrm{A}$ was being obnoxious and wanted to insult $\mathrm{B}$ for her ignorance, then he may use (15).

In the second scenario, A did not understand the content of the talk but thought that B understood it well. To inquire the content of the talk, it is more felicitous to use a wh-question like (15). Again, it is possible to use the $w h$-question+ho strategy in (16), but in a marked way. This time 
the markedness comes from the feeling that A was trying to get B to explain the content of the talk without admitting that $\mathrm{B}$ was in a privileged position to explain it.

Keoi gong me ne?

he say what WHQ

'What did he say?'

Wh-question

(Preferred in Scenario B: My friend was puzzled, too.)

(16) Keoi gong me ne ho?

he say what WHQ HO

'What did he say? Do you share the same question?'

(Preferred in Scenario A: My friend understood it.)

Wh-question + ho

\subsection{Synthesizing the two paradigms}

The properties of ho-questions discussed in the previous subsections raise two theoretically interesting questions. First, what does it mean for a particle to operate on an assertion or a question? Assertions and questions are speech act-level objects. Operating on these objects at the very least calls for a mechanism for manipulating speech acts. While the traditional view is that speech acts are inoperable, pragmatic objects, this view has been challenged in recent years, by scholars such as Krifka (2015), Davis (2009), and Heim et al. (2016). These scholars hold the view that speech acts should in principle be amenable to semantic operations just like other semantic objects. The fact that there are sentence-final particles operating on speech acts provides independent support for such a view.

Secondly, questions and assertions make different contributions to context, but ho indiscriminately operates on both types of speech acts, not minding their differences. Nonetheless, ho-questions have quite different interpretive properties depending on whether the embedded speech act is an assertion or a question. In the case of an assertion-ho-question, it asks for confirmation of the asserted content; in the case of a question-ho-question, it asks whether the addressee shares the question or not. Logically speaking, one could posit two instances of ho that embed assertions and questions, respectively. If we make this move, however, we miss capturing a strong intuition that native speakers of Cantonese have: ho-questions are really a uniform class and that's why the same particle is used to embed assertions and questions.

We argue in the rest of the paper that there is no need to posit two different ho's, as long as we take seriously the sentence-final particle stacking paradigm and treat ho as a higher-level question particle, one that embeds speech act rather than semantic content.

This way of cutting up the pie straightforwardly addresses the first question: ho may embed a question or an assertion because, as a speech act level question particle, it is in a position to do 
so. In addition, treating ho as a speech act level question particle buys us more than just a way to account for sentence-final particle stacking. It actually makes available a level, i.e., the speech act level, with which we can afford a unified semantics of ho in both the assertion embedding and question embedding contexts. We develop the concrete semantics of ho and a mechanism of speech act embedding in Section 3.

\section{Proposal}

We propose that ho embeds a speech act, which can be an assertion or a question. Following the dynamic semantics of discourse initiated in Farkas and Bruce (2009) and further developed in Rawlins (2010), Farkas and Roelofsen (2017) and Bledin and Rawlins (2017), speech acts are derived by combining speech act operators with corresponding semantic content. In section 3.1, we will lay out the formal preliminaries of the framework and define the speech act operators assert and quest. The speech act operators consist of two components: (a) an at-issue component, which instructs how a speaker updates the input context by acting on some semantic content; and (b) a non-at-issue component, which is a set of felicity conditions, which tests whether the speech act is felicitously performed. The semantics of ho is given in section 3.3. Briefly speaking, ho inherits the felicity condition of the speech act that it embeds, and generates a question for the addressee asking whether s/he is also able to felicitously perform the embedded speech act. This analysis can successfully capture the empirical patterns of ho, as demonstrated in section 3.3.1 and 3.3.2.

\subsection{Preliminaries}

Many studies have defined discourse contexts as a tuple consisting of different conversational components, like the Stalnakerian context set, a set of participants, commitment sets and so on (Gunlogson, 2001; Farkas and Bruce, 2009; Rawlins, 2010). Since not all of the conversational components are useful for our purpose, we define a context as a simple pair, consisting of a context set and a stack:

A context $c$ is a pair of $\left\langle\mathbf{c s}_{c}, \mathscr{T}_{c}\right\rangle$, where

a. $\quad \mathbf{c s}_{c} \subseteq W$ is a set of worlds (the context set)

b. $\quad \mathscr{T}_{c}$ is a stack of issues, i.e., a set of propositions.

Following Stalnaker $(1978,2002)$ and many others, the context set $\mathbf{c s}_{c}$ includes the possibilities that are compatible with what is known to the discourse participants for the purposes of the conversation. $\mathscr{T}_{c}$ is a stack of issues, i.e., a set of propositions, comparable to the Table component in Farkas and Roelofsen (2017) (cf. Farkas and Bruce, 2009; Malamud and Stepheson, 2015). The stack keeps a history of the utterances, i.e. the proposals for updating the context set, made by the discourse participants. ${ }^{6}$ The motivation for this component, due to Farkas and Bruce (2009), is

\footnotetext{
${ }^{6}$ Other formulations may involve more fine-grained structuring of the stack to separate assertions and questions, for
} example, Rawlins (2010), Bledin and Rawlins (2017). We adopt a simpler version for our purposes. 
that an assertion does not directly update the context set, but rather is a proposal to do so.

In this spirit, we define the speech act operator assert as shown below. It combines with a proposition and returns a context change potential, i.e., a function from an input context to an output context. The subscripts on the operator indicate the world parameter and the speaker parameter relative to which the speech act is evaluated.

$$
c+\text { assert }_{w, \mathbf{s}_{c}}(p)=\left\langle\mathbf{c s}_{c}, \operatorname{push}\left(\left\{\mathbf{c s}_{c} \cap\left\{w^{\prime} \mid p\left(w^{\prime}\right)\right\}\right\}, \mathscr{T}_{c}\right)\right\rangle, \text { defined only if }
$$

$\mathbf{S}_{c}$ believes that $p$ is true in $w$

push is a standard operation on stacks, formally defined as follows (see Farkas and Bruce (2009) for similar uses).

$$
\operatorname{push}(e, \mathscr{T})=e \cdot \mathscr{T} \text {, represents a new stack with } e \text { added to the top of } \mathscr{T} \text {. }
$$

According to (18), making an assertion involves pushing a proposal onto the stack. The proposal is modeled as a (singleton) set of contexts updated with the asserted proposition (cf. the projected set in Farkas and Bruce (2009)). In other words, an assertive update does not update the context set immediately, but rather makes a proposal pending the audience's response (confirm/reject). An assertive update is evaluated relative to two parameters, a possible world in which the speech act is defined and the author of the speech act.

This operator also comes packaged with an important felicity condition, which tracks the mental state of the speaker. Specifically, the felicity condition captures the intuition that a person appropriately asserts $p$ in $w$ only if they believes $p$ is true in $w$. Thus, an assertive update is felicitous only in worlds in which the speaker believes in the validity of $p$ and undefined otherwise. ${ }^{7}$

Moving on to questions, our questioning update is also formalized with use of a speech act operator-quest, defined as in (20). This operator takes a question $Q$ and returns a context change potential. In this paper, we follow Hamblin/Karttunen's approach (Hamblin, 1973; Karttunen, 1977) and assume that a question denotes a set of propositions. Each proposition in the set can potentially update the context set. Therefore, asking a question involves making a proposal that contains multiple potential updates. Then, the addressee answers the question by choosing one update from the proposal.

\footnotetext{
${ }^{7} \mathrm{We}$ are aware that this felicity condition makes a direct connection between true belief and the performance of an assertion, which cannot accommodate prevarication contexts (p.c. Manfred Krifka). In order to allow assertions in prevarication contexts, we can switch to a weaker felicity condition:
}

$$
c+\operatorname{assert}_{w, \mathbf{s}_{c}}(p)=\left\langle\mathbf{c s}_{c}, \operatorname{push}\left(\left\{\mathbf{c s}_{c} \cap\left\{w^{\prime} \mid p\left(w^{\prime}\right)\right\}\right\}, \mathscr{T}_{c}\right)\right\rangle, \text { defined only if }
$$

$\mathbf{s}_{c}$ wants her fellow discourse participants to believe that she believes $p$ is true in $w$

This switch has no bearing on our central thesis, and hence we use the stronger version for the sake of simplicity. 


$$
c+\text { quest }_{w, \mathbf{s}_{c}}(Q)=\left\langle\mathbf{c s}_{c}, \operatorname{push}\left(\left\{\mathbf{c s}_{c} \cap\left\{w^{\prime} \mid p\left(w^{\prime}\right)\right\} \mid p \in Q\right\}, \mathscr{T}_{c}\right)\right\rangle, \text { defined only if }
$$

$\mathbf{s}_{c}$ does not know the answer to $Q$ in $w$

The questioning update has two felicity conditions. Presumably, a questioner can appropriately ask $Q$ only if she does not already know the answer to $Q .{ }^{8}$

\subsection{Formalizing Cantonese sentence-final particles}

Before laying out the formal analysis of ho, we will apply the formal tools defined in the last section to distinguish declaratives and questions from Cantonese. Consider (21).

$$
\begin{aligned}
& \text { Aaman sik haa gaa. } \\
& \text { Aaman eat shrimp ASSERT } \\
& \text { 'Aaman eats shrimp.' }
\end{aligned}
$$

We assume that the sentence final particle gaa lexically encodes assert. The declarative sentence, then, has the following translation:

$$
\begin{aligned}
& c+\text { assert }_{w, \mathbf{s}_{c}}(\llbracket \text { Aaman eat shrimp } \rrbracket)= \\
& \left.\qquad \mathbf{c s}_{c}, \text { push }\left(\left\{\mathbf{c s}_{c} \cap\left\{w^{\prime} \mid \begin{array}{l}
\text { Aman eat } \\
\text { shrimp in } w^{\prime}
\end{array}\right\}\right\}, \mathscr{T}_{c}\right)\right\rangle \\
& \text { defined only if } \mathbf{s}_{c} \text { believes Aaman eats shrimp in } w
\end{aligned}
$$

Accordingly, (21) means that the speaker proposes in world $w$ to update the input context with the proposition denoted by Aaman eats shrimp. If the assertion is appropriately made in the world, the speaker must believe Aaman eats shrimp.

Turning to questions, we take (23) as an example. Like gaa, the question particle ne is also assumed to contain quest in its lexical semantics. Assuming that the possible answers to the question are Aaman eats shrimp and Waazai eats shrimp, we translate the question as (24). ${ }^{9}$

$$
\begin{aligned}
& \text { Bingo sik haa ne? } \\
& \text { who eat shrimp WHQ } \\
& \text { 'Who eats shrimp?' }
\end{aligned}
$$

\footnotetext{
${ }^{8}$ Note that these felicity conditions can be suspended in exam/quiz contexts and other non-standard scenarios like the rhetorical use of questions, and we have no new insight to offer regarding how suspension is allowed.

${ }^{9}$ For simplicity, we assume an unstructured domain of possible answers. However, our analysis is compatible with a more structured domain containing pluralities, as argued by Dayal (1996).
} 


$$
\begin{aligned}
& c+\text { quest }_{w, \mathbf{s}_{c}}(\llbracket \text { who eats shrimp } \rrbracket)= \\
& \qquad\left\langle\mathbf{c s}_{c}, \text { push }\left(\left\{\begin{array}{l}
\mathbf{c s}_{c} \cap\left\{w^{\prime} \mid \text { Aaman eat shrimp in } w^{\prime}\right\} \\
\mathbf{c s}_{c} \cap\left\{w^{\prime} \mid \text { Waazai eat shrimp in } w^{\prime}\right\}
\end{array}\right\}, \mathscr{T}_{c}\right)\right\rangle
\end{aligned}
$$

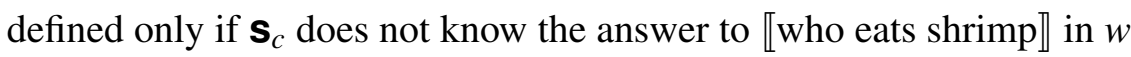

With the use of question (23), the questioner proposes that the input context can be updated with the proposition denoted by Aaman eats shrimp or the one denoted by Waazai eats shrimp. If the question is appropriately asked, the questioner must want to know its answer, as dictated by its felicity condition.

A note for clarification before we move on: although we take SFPs to lexically encode speech act operators like assert and quest, we by no means imply a unique mapping between a SFP and a speech act operator. As mentioned in Section 2.1, a sentence may be interpreted as a question or an assertion even without any SFP. Additionally, there is more than one assertion particle and question particle in Cantonese. In fact, in Section 4, we discuss two variants of the quest operator with slightly different felicity conditions.

\subsection{Semantics of ho}

Armed with these definitions, we can now present our analysis of ho. Consider the definition in (25), in which $A$ is a variable for the speech act embedded by ho.

$$
\begin{aligned}
c+h o(A)_{w, \mathbf{s}_{c}, \mathbf{a}_{c}=}= & \left\langle\mathbf{c s}_{c}, \text { push }\left(\left\{\begin{array}{l}
\mathbf{c s}_{c} \cap\left\{w^{\prime} \mid A_{w^{\prime}}, \mathbf{a}_{c} \text { is defined in } w^{\prime}\right\}, \\
\mathbf{c s}_{c} \cap\left\{w^{\prime} \mid A_{w^{\prime}, \mathbf{a}_{c}} \text { is undefined in } w^{\prime}\right\}
\end{array}\right\}, \mathscr{T}_{c}\right)\right\rangle \\
& \text { defined only if } A_{\mathbf{s}_{c}} \text { is defined in } w
\end{aligned}
$$

This definition says: ho takes the speech act $A$ as its argument; then, it forms a new question asking whether the addressee $\mathbf{a}_{c}$ can felicitously perform $A$ or not; this new question is formalized as a set of two possible updates; one is the intersection of the context set $\mathbf{c s}_{c}$ and the worlds in which $\mathbf{a}_{c}$ 's performance of $A$ is defined, i.e., updating $\mathbf{c s}_{c}$ with the proposition that $\mathbf{a}_{c}$ felicitously performs $A$; the other is the intersection of $\mathbf{c s}_{c}$ and the worlds in which $\mathbf{a}_{c}$ 's performance of $A$ is undefined, i.e., updating $\mathbf{C s}_{c}$ with the proposition that $\mathbf{a}_{c}$ does not felicitously perform $A$; finally, the composition of $h o$ and $A$ returns a new speech act, which is defined only if the speaker $\mathbf{S}_{c}$ can felicitously perform $A$. Thus, ho informally expresses two facts: i) that the speaker can felicitously perform a speech act and, ii) they are asking whether the addressee can felicitously perform the same speech act. 


\subsubsection{Assertion $+h o$}

Let's use the familiar data point below as a concrete example to elaborate how the definition in (25) captures the Cantonese facts.

$$
\begin{aligned}
& \text { Aaman sik haa gaa ho? } \\
& \text { Aaman eat shrimp ASSERT HO } \\
& \text { 'Aaman eats shrimp. Right?' }
\end{aligned}
$$

In this example, ho is attached to the assertion marked by gaa. With use of (25), we translate the sentence as follows:

$$
\begin{gathered}
c+h o(\text { assert }(\llbracket \text { Aaman eat shrimp } \rrbracket))_{w, \mathbf{s}_{c}, \mathbf{a}_{c}=} \\
\left\langle\mathbf{c s}_{c}, \text { push }\left(\left\{\begin{array}{l}
\mathbf{c s}_{c} \cap\left\{w^{\prime} \mid \text { assert }_{w^{\prime}}, \mathbf{a}_{c}(\llbracket \text { Aaman eat shrimp } \rrbracket) \text { is defined in } w^{\prime}\right\}, \\
\mathbf{c s}_{c} \cap\left\{w^{\prime} \mid \text { assert }_{w^{\prime}}, \mathbf{a}_{c}(\llbracket \text { Aaman eat shrimp } \rrbracket) \text { is undefined in } w^{\prime}\right\}
\end{array}\right\}, \mathscr{T}_{c}\right)\right\rangle \\
\text { defined only if } \text { assert }_{w, \mathbf{s}_{c}}(\llbracket \text { Aaman eat shrimp } \rrbracket) \text { is defined in } w
\end{gathered}
$$

According to the felicity condition of assert, as in (18), if it is defined for $\mathbf{a}_{c}$ to assert Aaman eats shrimp, then $\mathbf{a}_{c}$ believes Aaman eats shrimp. Conversely, if it is undefined for $\mathbf{a}_{c}$ to assert Aaman eats shrimp, then $\mathbf{a}_{c}$ does not believe Aaman eats shrimp. Based on these deductions, we can predict the possible responses to (26), which are described in section 2.2 and repeated here:

$$
\begin{aligned}
& \begin{array}{ll}
\text { a. Hai aa. } & \text { b. Mhai aa } \\
\text { yes ASSERT } & \text { no ASSERT }
\end{array} \\
& \text { 'Yes, he does.' 'No, he doesn't' }
\end{aligned}
$$
c. jo mzi wo
I not.know SFP
'I don't know.'

When the addressee responds with (28a), it indicates that they also believe that Aaman eats shrimp. If the addressee does not share this belief with the speaker, they may know that Aaman does not eat shrimp or they may be ignorant about the propositional content. In the former case they can use (28b) to answer the question, while in the latter case they can use (28c).

In Section 2.2, we noted that an assertion-ho question bears a bias towards the asserted proposition, making it less felicitous than a default polar question (with $\mathrm{maa}$ ) in a neutral context (see example (7) and (8)). The felicity condition of attaching ho to an assertion can capture the presence of this bias. In (27), if the speaker can felicitously assert that Aaman eats shrimp, then they believe that this proposition is true. It then entails that the speaker does not believe that the counterpart of the asserted proposition Aaman does not eat shrimp is true. A consequence of this belief in the validity of $p$ over $\neg p$ results in a bias towards $p$ over $\neg p .^{10}$

\footnotetext{
${ }^{10}$ Without going into a lot of detail, the definition of bias we adopt for our purposes here is one that treats the asserted nucleus of a question as more salient in the speaker's doxastic domain than its counterpart. See Roelofsen and van Gool (2010), Biezma and Rawlins (2012), Krifka (2015), Xu (2017), Bhadra (2017) among others.
} 


\subsubsection{Question + ho}

We now demonstrate how the proposed analysis can account for the patterns with questions embedded by ho. Consider (29), repeated from (9):

Bingo sik haa ne ho?

who eat shrimp WHQ HO

'Who eats shrimp? Do you share the same question?'

Wh-question + ho

Based on our assumption that ne lexically encodes quest, we translate the question in (9) as follows:

$$
\begin{aligned}
& c+h o(\text { quest }(\llbracket \text { who eat shrimp } \rrbracket))_{w, \mathbf{s}_{c}, \mathbf{a}_{c}}= \\
& \left\langle\mathbf{c s}_{c}, \text { push }\left(\left\{\begin{array}{l}
\mathbf{c s}_{c} \cap\left\{w^{\prime} \mid \text { quest }_{w^{\prime}}, \mathbf{a}_{c}(\llbracket \text { who eats shrimp } \rrbracket) \text { is defined in } w^{\prime}\right\}, \\
\mathbf{c s}_{c} \cap\left\{w^{\prime} \mid \text { quest }_{w^{\prime}, \mathbf{a}_{c}}(\llbracket \text { who eats shrimp } \rrbracket) \text { is undefined in } w^{\prime}\right\}
\end{array}\right\}, \mathscr{T}_{c}\right)\right\rangle \\
& \text { defined only if quest }{ }_{w, \mathbf{s}_{c}}(\llbracket \text { who eats shrimp } \rrbracket) \text { is defined in } w
\end{aligned}
$$

Accordingly, the result of uttering (9) is to push onto $\mathscr{T}_{c}$ the issue of whether or not the addressee $\mathbf{a}_{c}$ can felicitously ask the embedded question who eats shrimp. It comes packaged with the felicity condition that the speaker $\mathbf{S}_{c}$ can ask the embedded question felicitously.

In the scenario where $\mathbf{a}_{c}$ can felicitously perform the question act, it entails that they are ignorant of the answer. In this scenario, $\mathbf{a}_{c}$ may choose an answer like (31a), repeated from (14a), to indicate that the question act is defined for them. Alternatively, in a scenario where $\mathbf{a}_{c}$ cannot felicitously perform the question act, it entails just the opposite, namely, that they know the answer to the question. If this is indeed the case, then $\mathbf{a}_{c}$ may choose to answer the embedded question with something like (31b), repeated from (14b).
a. Hai lo.
b. Aaman lo.
yes SFP
Aaman SFP
'Yes.'
'Aaman.'

Another way for the addressee to indicate that she may felicitously perform a question act is to acknowledge that she is also ignorant about the answer to the question, just like the speaker is. In this case, an answer like (32a), repeated from (14c), may be used and has the same effect as (31a). However, due to the binary nature of the choice, the addressee will never be ignorant about her ability to perform such a question act. In other words, the addressee either can ask the question or cannot ask the question. For this reason, an answer like (32b), which lacks the additive particle dou and hence indicates ignorance towards the matrix question, is judged to be infelicitous. 

a. Ngo dou mzidou wo.
I also not.know SFP
'I also don't know.'

b. \#Ngo mzidou wo.
I not.know SFP
'I don't know.'

The present analysis can also capture the felicity of Q-ho questions. As described in section 2.3, a Q-ho question is appropriate when the speaker does not expect the addressee to know the answer to the question embedded by ho. The relevant example is repeated below:

Scenario: A famous scientist gave a talk on astrophysics. A, as a linguist, could not follow the talk. A's friend B was a poet, and it seemed to A that B did not understand the talk either (see Figure 1).
Keoi gong me ne ho?
he say what WHQ HO
'What did he say? Do you share the
same question?'
(34) \#Keoi gong me ne? he say what WHQ 'What did he say?'

In the scenario, the ho-Q question, rather than the ordinary wh-question, is more felicitous. According to Farkas and Bruce (2009), the context state following an ordinary question is inquisitive with respect to the denotation of the sentence radical that is pushed onto $\mathscr{T}_{c}$. For example, uttering (34) indicates that the speaker would like to update the context in one of the relevant ways, i.e., intersecting $\mathbf{c s}_{c}$ with different propositions contained in the set denoted by what did he say, but s/he is not sure which update matches the fact in the actual world. Therefore, in order to successfully update the context, the speaker expects the addressee to pick out one of the possible updates. In other words, the addressee is expected to provide an answer to the question. However, the given scenario implies that the speaker does not believe the addressee knows the answer. As a result, asking (34) is not felicitous.

By contrast, the speaker's inquiry is transformed when the question is embedded under $h o$, as in (33). According to the definition of ho, this question can be translated as:

$$
\begin{aligned}
& c+h o(\text { quest }(\llbracket \text { what did he say } \rrbracket))_{w, \mathbf{s}_{c}, \mathbf{a}_{c}}= \\
& \left\langle\mathbf{c s}_{c}, \text { push }\left(\left\{\begin{array}{l}
\mathbf{c s}_{c} \cap\left\{w^{\prime} \mid \text { quest }_{w^{\prime}, \mathbf{a}_{c}}(\llbracket \text { what did he say } \rrbracket) \text { is defined in } w^{\prime}\right\}, \\
\mathbf{c s}_{c} \cap\left\{w^{\prime} \mid \text { quest }_{w^{\prime}, \mathbf{a}_{c}}(\llbracket \text { what did he say } \rrbracket) \text { is undefined in } w^{\prime}\right\}
\end{array}\right\}, \mathscr{T}_{c}\right)\right\rangle \\
& \text { defined only if quest } \text { ques }_{w}(\llbracket \text { what did he say } \rrbracket) \text { is defined in } w
\end{aligned}
$$

In this case, the speaker intends to update the context with one of the two possible mental states of the addressee's: either the addressee can ask what did he say or s/he cannot. The former entails the addressee's ignorance towards the question, while the latter entails the addressee's knowledge regarding the question. Therefore, the speaker does not need to expect the addressee to know the answer to the embedded question. In fact, if the speaker does have such a belief, she would use (34) instead of (33). 


\section{Other question particles}

So far, we have discussed the question particle ne, and its interaction with ho. As mentioned in section 2.1, Cantonese has other question particles. For example, to indicate a polar question, the polar question particle maa may be used (36). There is also a particle $a a$, which can be used in wh-questions and alternative questions (37).

$$
\begin{aligned}
& \text { Aaman sik haa maa? } \\
& \text { Aaman eat shrimp POLQ } \\
& \text { 'Does Aaman eat shrimp?' }
\end{aligned}
$$

Polar question particle maa

Lei-go hai mei jisi aa? this-Cl is what mean AA 'What does this mean?'

Wh/Alternative-question particle aa

What is interesting about these particles is that a special context is required to use the questions resulting from stacking them under ho, or else a pragmatically marked flavor arises. The natural context to use questions like (38a) and (38b) is a 'switch addressee' context. In such a context, the question embedded by ho is directed to an addressee but the whole ho-question is directed to a different addressee. If not used in such a context, (38a) and (38b) are very marked, and almost seem like an indirect and somewhat pretentious way to get the addressee to provide an answer to the embedded question. In this paper, we do not formally deal with the 'switch addressee' context (but see footnote 12 for an informal discussion). However, we would like to suggest a way to derive the markedness of these questions when they are not used in a 'switch addressee' context.

a. $m a a+h o$

Aaman sik haa maa ho?

Aaman eat shrimp POLQ HO

'Does Aaman eat shrimp? Do you share the same question?'

Marked: addressed to the same addressee

Unmarked: addressed to different addressees

b. $a a+h o^{11}$

Bingo sik haa aa ho?

who eat shrimp AA HO

'Who eats shrimp? Do you share the same question?'

Marked: addressed to the same addressee

Unmarked: addressed to different addressees

A related observation is that maa and $a a$ may not be used when there is no addressee at all, but ne is

\footnotetext{
${ }^{11}$ This question is acceptable when the embedded question is used rhetorically. A related observation, due to Lam (2014), is that ho may stack on the biased polar question particle me. We leave rhetorical questions feeding ho for future studies.
} 
fine in such a context. We take this to suggest that maa and $a a$ have an additional felicity condition requiring the obligatory presence of an addressee who is expected to answer the question. It is this additional felicity condition that gives rise to the pragmatic flavor. To see this, let us define the quest operator corresponding to $a a$ ( $m a a$ can be defined in a similar manner). It is identical to $n e$ except for an extra felicity condition in (b).

$$
\begin{aligned}
c+\text { quest }_{w, \mathbf{s}_{c}, \mathbf{a}_{c}}^{\mathrm{aa}}(Q)= & \left\langle\mathbf{c s}_{c}, \boldsymbol{p u s h}\left(\left\{\mathbf{c s}_{c} \cap\left\{w^{\prime} \mid p\left(w^{\prime}\right)\right\} \mid p \in Q\right\}, \mathscr{T}_{c}\right)\right\rangle, \text { defined only if } \\
& \text { a. } \mathbf{s}_{c} \text { does not know the answer to } Q \text { in } w \\
& \text { b. } \mathbf{s}_{c} \text { believes that } \mathbf{a}_{c} \text { can answer } Q \text { in } w
\end{aligned}
$$

quest $^{\mathrm{aa}}$ leads to the same inquisitive context as quest ${ }^{\mathrm{ne}}$ does, but it has one more felicity conditionthe speaker believes that the addressee knows the answer to the embedded question. Accordingly, using an $a a$-question is only appropriate if the question is directed to a person that the speaker thinks is able to resolve the question. As a result, an $a a$-question can never be self-directed.

Combining an $a a$-question with ho results in an odd question. Take (38b) as an example. The denotation of this sentence is represented as (40).

$$
\begin{gathered}
c+h o\left(\text { quest }^{\mathrm{aa}}(\llbracket \text { who eats shrimp } \rrbracket)\right)_{w, \mathbf{s}_{c}, \mathbf{a}_{c}}= \\
\left\langle\mathbf{c s}_{c}, \text { push }\left(\left\{\begin{array}{c}
\mathbf{c s}_{c} \cap\left\{w^{\prime} \mid \text { quest }_{w^{\prime}, \mathbf{a}_{c}, \mathbf{s}_{c}}^{\mathrm{aa}}(\llbracket \text { who eats shrimp } \rrbracket) \text { is defined in } w^{\prime}\right\}, \\
\mathbf{c s}_{c} \cap\left\{w^{\prime} \mid \text { quest }_{w^{\prime}, \mathbf{a}_{c}, \mathbf{s}_{c}}^{\mathrm{aa}}(\llbracket \text { who eats shrimp } \rrbracket) \text { is undefined in } w^{\prime}\right\}
\end{array}\right\}, \mathscr{T}_{c}\right)\right\rangle \\
\text { defined only if quest } \mathbf{w}_{w, \mathbf{s}_{c}, \mathbf{a}_{c}}^{\mathrm{aa}}(\llbracket \text { who eats shrimp } \rrbracket) \text { is defined in } w
\end{gathered}
$$

(40) updates the context by pushing onto $\mathscr{T}_{c}$ a question that can be paraphrased as: can the addressee $\boldsymbol{a}_{c}$ perform the aa-question felicitously or not. We argue that the addressee would never pick the positive member in the set, because the positive member represents a set of felicity conditions that contradict the felicity conditions of accepting (40). Suppose to the contrary that (40) is accepted and $\mathbf{a}_{c}$ picks the positive member, namely, that it is defined for $\mathbf{a}_{c}$ to perform the $a a$ question. What this implies is that $\mathbf{a}_{c}$ is ignorant of the answer to the question who eats shrimp and believes that their addressee (i.e., the speaker $\mathbf{S}_{c}$ ) can provide the answer. This gives rise to a contradiction. The whole update characterized by (40) is defined only if $\mathbf{S}_{c}$ is ignorant of the answer to who eats shrimp and expects $\mathbf{a}_{c}$ to provide the answer. So, $\mathbf{a}_{c}$ cannot reasonably believe that $\mathbf{S}_{c}$ can provide the answer to the $a a$-question, prohibiting $\mathbf{a}_{c}$ from picking the positive member. ${ }^{12}$

\footnotetext{
${ }^{12}$ In a 'switch-addressee' context, the additional felicity condition is not problematic because the speaker now only believes that the addressee of the embedded $a a$-question can provide an answer to the question. As a consequence, for the addressee of the ho-question to felicitously ask the $a a$-question, they only need to believe that the addressee of the embedded question, which is no longer the speaker, knows the answer to the aa-question. We have to leave the discussion of the 'switch-addressee' context informal primarily due to the lack of space for developing a mechanism for changing the addressee parameter of a speech act operator.
} 
Since one of the proposed updates in (40) is defunct and will never be picked by the addressee, the ho-question is not a well-defined inquisitive update. Rather, it bears a pragmatic effect similar to that of a rhetorical question, namely, only one of the proposed updates is consistent with the context. In this case, the only plausible update is that asking the same $a a$-question is undefined for the addressee.

If we unpack what it means for the addressee to pick the undefined option, we will see why the whole ho-question is often used to coax the addressee into actually answering the embedded $a a$ question. First, the addressee cannot felicitously ask the $a a$-question for an obvious reason, namely, that their addressee (i.e., the speaker of (40)) cannot provide an answer to the question. It is not informative for the speaker. This is because if a speaker utters (40), he has already indicated that he does not know the answer to the embedded $a a$-question.

Second, if the addressee knows how to answer the $a a$-question, it is also infelicitous for them to use this question. In this case, assuming a cooperative conversational partner, the speaker expects the addressee to answer the $a a$-question directly. As a result, the speaker can use the $a a$-ho-question as an indirect way to elicit an answer to the embedded $a a$-question.

\section{Conclusion}

This paper pursued the claim that the grammatical embeddability of speech act operators under higher operators is based on a system of compositional semantics at the speech act level. Basing the discussion on Cantonese ho, we argued that ho operates on speech acts and returns a higher level speech act that has the effect of asking the addressee if they would like to perform the same speech act as the speaker. The contribution of this particle is modeled in an update semantics, whereby speech act operators have two components: an overt instruction regarding how to update the input context, as well as a mechanism of checking whether the speech act is felicitously performed. In future research, we seek to uncover such particles in other languages with a rich inventory of sentence-final particles, as well as to extend the speech act embedding mechanism developed here to account for other speech act phenomena.

\section{References}

Bhadra, D. (2017). Evidentiality and questions: Bangla at the Interfaces. PhD thesis, Rutgers University, New Brunswick, NJ.

Biezma, M. and Rawlins, K. (2012). Responding to alternative and polar questions. Linguistics and Philosophy, 35:361-406.

Bledin, J. and Rawlins, K. (2017). What if questions. Johns Hopkins University. Manuscript.

Cheung, S. H.-N. (1972). Xianggang Yueyu Yufa Yanjiu [A Study of Hong Kong Cantonese Grammar]. University of Hong Kong, Hong Kong.

Davis, C. (2009). Decisions, dynamics and the Japanese particle yo. Journal of Semantics, 26:329-336.

Davis, C. (2011). Constraining interpretation: Sentence final particles in Japanese. PhD thesis, University of Massachusetts Amherst, Amherst, Massachusetts.

Dayal, V. (1996). Locality in Wh-Quantification: Questions and Relative Clauses in Hindi. Kluwer Academic Press, 
Dordrecht.

Farkas, D. and Bruce, K. (2009). On reacting to assertions and polar questions. Journal of Semantics, 27:81-118.

Farkas, D. and Roelofsen, F. (2017). Division of labor in the interpretation of declaratives and interrogatives. Journal of Semantics, 34:237-289.

Frege, G. (1956). The thought: A logical inquiry. Mind, 56(259):289-311.

Fung, R. S.-Y. (2000). Final Particles in Standard Cantonese : Semantic extension and pragmatic inference. PhD thesis, The Ohio State University, Columbus, Ohio.

Gunlogson, C. (2001). True to form: Rising and falling declaratives as questions in English. Routledge, London.

Hamblin, C. (1973). Questions in Montague grammar. Foundations of Language, 10(1):41-53.

Heim, J., Keupdjio, H., Lam, Z. W.-M., Osa-G' omez, A., Thoma, S., and Wiltschko, M. (2016). Intonation and particles as speech act modifiers: A syntactic analysis. Studies in Chinese Linguistics, 37:109-129.

Karttunen, L. (1977). Syntax and semantics of questions. Linguistics and Philosophy, 1(1):3-44.

Krifka, M. (2015). Bias in Commitment Space Semantics: Declarative questions, negated questions and question tags. In D’Antonio, S. and Moroney, M., editors, Proceedings of Semantics and Linguistic Theory 25, pages 328-345, Ithaca, NY. Cornell University.

Lam, Z. W.-M. (2014). A complex ForceP for speaker- and addressee-oriented discourse particles in cantonese. Studies in Chinese Linguistics, 35:61-80.

Luke, K.-K. (1990). Utterance particles in Cantonese conversation. John Benjamins Publishing Company, Amsterdam.

Malamud, S. and Stepheson, T. (2015). Three ways to avoid commitments: Declarative force modifiers in the conversational scoreboard. Journal of Semantics, 32:275-311.

Matthews, S. and Yip, V. (2011). Cantonese: A Comprehensive Grammar. Routledge, London \& New York, 2nd edition edition.

Rawlins, K. (2010). Conversational backoff. In Li, N. and Lutz, D., editors, Proceedings of Semantics and Linguistic Theory 20, pages 347-365, Ithaca, NY. Cornell University Press.

Roelofsen, F. and van Gool, S. (2010). Disjunctive questions, intonation and highlighting. In Aloni, M., Bastiaanse, H., de Jager, T., and Schultz, K., editors, Logic, Language, and Meaning: Selected Papers from the 17th Amsterdam Colloquium, pages 384-394, Berlin Heidelberg. Springer.

Stalnaker, R. (1978). Assertion. In Cole, P., editor, Syntax and Semantics 9: Pragmatics, pages 315-322. Academic Press, New York.

Stalnaker, R. (2002). Common ground. Linguistics and Philosophy, 25:701-721.

Sybesma, R. and Li, B. (2006). The dissection and structural mapping of Cantonese sentence final particles. Lingua, 117:1739-1783.

Tang, S.-W. (2015). A generalized syntactic schema for utterance particles in Chinese. Lingua Sinica, 1:3.

Wakefield, J. C. (2011). The English equivalents of Cantonese sentence-final particles. PhD thesis, The Hong Kong Polytechnic University, Hong Kong.

Wiltschko, M. (2017). Ergative constellations in the structure of speech acts. In Coon, J., Massam, D., and Travis, L. d., editors, The Oxford Handbook of Ergativity, pages 419-442. Oxford University Press.

Xu, B. (2017). Question Bias and Biased Question Words in Mandarin, German and Bangla. PhD thesis, Rutgers University, New Brunswick, NJ. 